\title{
Cell cycle of transdifferentiating supporting cells in the basilar papilla
}

\author{
Yehoash Raphael *, Henry J. Adler, Yu Wang, Peter A. Finger \\ Kresge Hearing Research Institute, The University of Michigan, 1301 East Ann Street, Ann Arbor, MI 48109-0506, USA
}

(Received 5 April 1994; revised 8 June 1994; accepted 30 June 1994)

\begin{abstract}
Mitosis of supporting cells has been shown to contribute to the cellular repopulation of the basilar papilla after acoustic trauma. In the present work we report data obtained with light and transmission electron microscopy after acoustic trauma in chicks. We report changes that occur in cell shape, surface morphology, intercellular junctions, nuclear shape and location, and cytoplasmic organization of supporting cells after trauma. The findings strongly suggest that supporting cells transdifferentiate and that the proliferative pattern is similar to interkinetic nuclear migration, as previously shown in the developing neural tube and basilar papilla. S-phase nuclei were positioned adjacent to the basement membrane, suggesting that interaction with the extracellular matrix may occur during the cell cycle. Supporting cells divided with the long axis of the spindle parallel to the reticular lamina and displayed no signs of intercellular communication during mitosis. This suggested to us that the fate of the progeny cells is determined prior to mitosis and that the progeny may be of identical phenotypic fate. Dividing cells had a smooth apical surface. The smooth surface may provide a marker to help identify dividing cells with scanning electron microscope analysis.
\end{abstract}

Keywords: Basilar papilla; Noise; Chick; Mitosis; TEM; Regeneration

\section{Introduction}

Several patterns of cellular proliferation and differentiation have been found in different tissues during development and regeneration. In most epithelial tissues, cell renewal is based on proliferation of basal (stem) cell populations, as in the epidermis, several types of glands and the olfactory neuroepithelium (Leblond and Walker, 1956; Graziadei and Monti Graziadei, 1985). Tissues that can regenerate despite the absence of stem cells are rare. One example is the retina of fish and embryonic amphibian and chick, where the retinal pigment epithelium can transdifferentiate into sensory retinal cells (Stone, 1950; Stroeva and Mitashov 1983).

In the auditory epithelium of birds no stem cells

\footnotetext{
Presented, in part, at the 17th Midwinter Meeting of the Association for Research in Otolaryngology, St. Petersburg, Florida, February 1994

* Corresponding author. Fax: 313 764-0014; e-mail: Yehoash. Raphael $@$ Med.Umich.Edu.
}

have been found. The only two types of epithelial cells that have been described in the avian basilar papilla are hair cells and supporting cells (Jahnke et al., 1969; Takasaka and Smith, 1971; Hirokawa, 1978; Tanaka and Smith, 1978). The apical membranes of hair cells and supporting cells in the basilar papilla create an orderly cellular mosaic called the reticular lamina which borders the endolymphatic lumen. The basal end of supporting cells extends from the luminal surface to contact the basement membrane, whereas hair cells, which are shorter, lack basement membrane contacts.

Despite the absence of stem cells, the avian basilar papilla can regenerate after acoustic trauma (Cotanche, 1987a). Findings that supporting cells in the basilar papilla can divide after acoustic trauma (Raphael, 1992) have provided strong evidence that transdifferentiation of supporting cells leads to repopulation of the epithelium. The source and nature of the signals that regulate the spatial-temporal sequence of transdifferentiation are yet to be identified. However, studies on development (Katayama and Corwin, 1993) and regeneration of the basilar papilla (Raphael, 1992; Stone and Cotanche, 1994) have suggested that the pattern of 

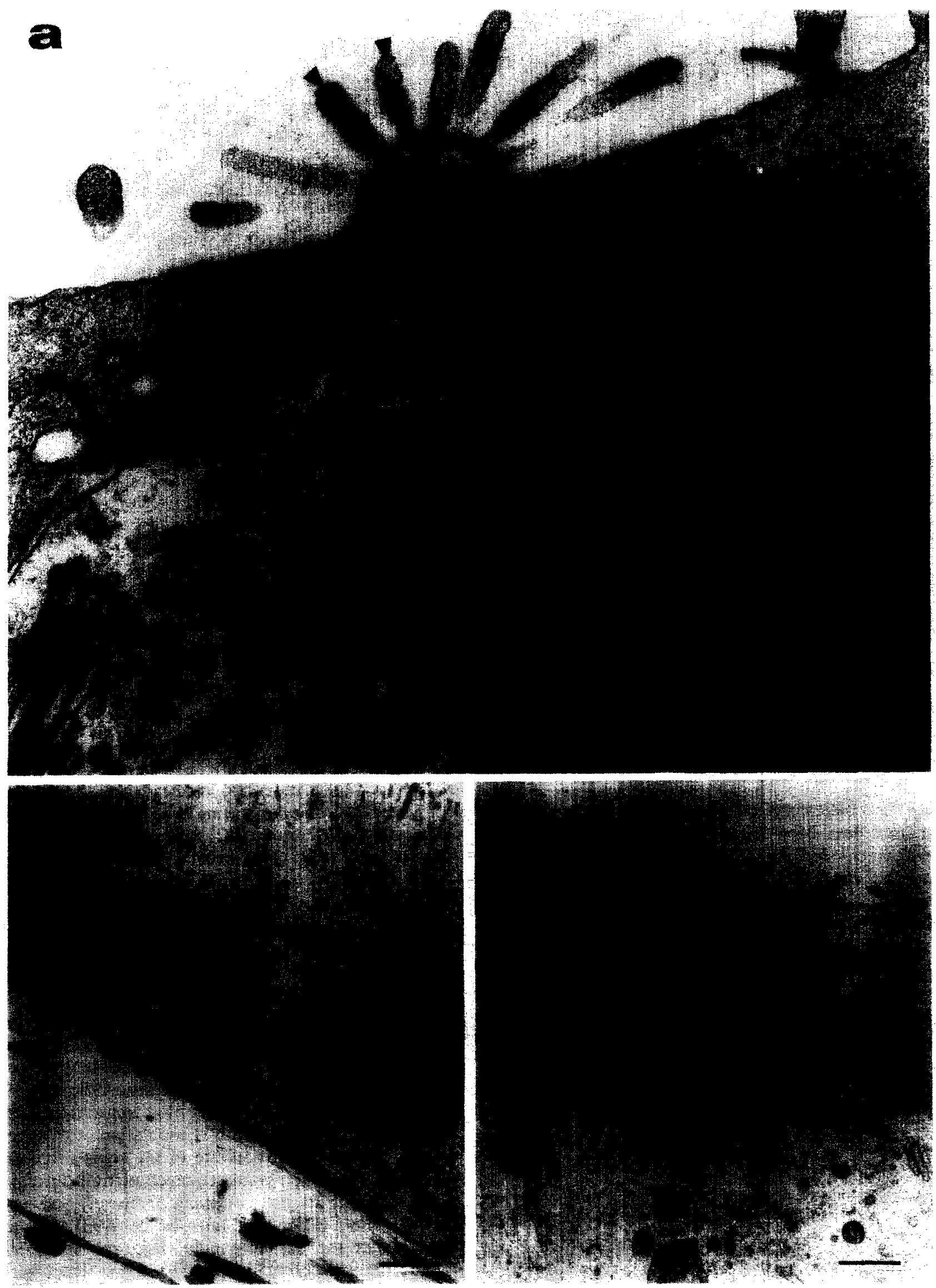
cell division is generally similar to that described in the neural tube during embryonic neurogenesis, namely, interkinetic nuclear migration (Sauer, 1935).

In order to better understand the signaling that regulates transdifferentiation, it is necessary to describe the changes that occur in cell shape and intercellular communication in the basilar papilla after trauma. Characterization of the relationship of cells with the basement membrane, neural tissue, luminal surface and other epithelial cells may be instrumental in identification of potential sources for transdifferentiationrelated signals. The purpose of this work was to characterize the structural changes that occur in supporting cells during the early response to acoustic trauma. Of particular interest were cell shape, luminal and basement membrane contacts, basal body location and orientation, cell volume, apical junctional complexes, basolateral intercellular communication and cytoskeletal organization. We overstimulated the auditory epithelium with noise and used transmission electron microscopy (TEM), a BrdU assay and DNA-specific labeling to determine ultrastructure and identify newly synthesized DNA, respectively. The results show that basement membrane components, neighboring supporting cells and surviving hair cells may all be involved in signaling for regulation of transdifferentiation.

\section{Materials and Methods}

Sixteen chicks (10-14 days old) were divided into 3 groups: group I ( 4 animals) for TEM analysis, group II (4 animals) for BrdU experiments and group III (2 animals) for DNA-specific labeling. All chicks were exposed to an octave band noise with a center frequency of $1.5 \mathrm{kHz}, 115 \mathrm{~dB}$ SPL for $4 \mathrm{~h}$, and killed 2 days after the exposure. The inner ears were fixed in $2.5 \%$ glutaraldehyde in $0.15 \mathrm{M}$ cacodylate buffer, $\mathrm{pH}$ 7.35 (group I) or $2 \%$ paraformaldehyde in $0.15 \mathrm{M}$

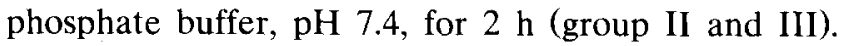
Group II chicks were injected once with BrdU (25 $\mathrm{mg} / \mathrm{kg}$ ) before the exposure, then again at 4 and $12 \mathrm{~h}$ post-exposure. Two control (unexposed) chicks were used for each of the three groups.

TEM samples were postfixed in $1 \%$ osmium tetroxide for $1 \mathrm{~h}$, dehydrated in alcohol and embedded in
Epon 812. Semi-thin sections were obtained with glass knives, mounted on microscope slides and stained with toluidine blue. Thin sections were cut with a diamond knife on a Reichert-Jung Ultracut E ultramicrotome, stained with uranyl acetate and lead citrate, and analyzed in a Jeol JEM 1200 EX electron microscope.

BrdU-specific antibodies were used to detect newly synthesized DNA. Whole mounts of the basilar papilla were permeabilized with Triton $\mathrm{X}-100$ and the DNA was denatured in $2 \mathrm{~N} \mathrm{HCl}$ for $30 \mathrm{~min}$. The samples were incubated with BrdU-specific monoclonal primary antibody solution (Sigma), followed by peroxidase-DAB processing, using the Vectastain ABC Kit from Vector Laboratories (Burlingame, CA). After completion of staining, the samples were lightly osmicated, embedded in Epon, and analyzed with light microscopy (LM). The plastic blocks were then sectioned for TEM.

For DNA-specific labeling we used whole mounts of the basilar papilla, labeled with Hoechst 33258 (Sigma), as previously described (Raphael, 1992). This method enabled us to observe all DNA in the tissue, in nuclei or in chromosomes of dividing cells.

The care and use of the animals in this study were approved by ULAM, the University committee on use and care of animals at the University of Michigan.

\section{Results}

Two days after noise exposure, a distinctive lesion was found in the basilar papilla. The center of the lesion was in the short hair cell area, 1-2 $\mathrm{mm}$ from the proximal end of the basilar papilla. The lesion involved hair cell loss and supporting cell expansion, primarily in the short hair cell area, as previously described with a similar trauma (Raphael, 1992, 1993; Stone and Cotanche, 1994).

\section{Supporting cells in regions without hair cell loss}

Supporting cells that were located between surviving hair cells often displayed a normal apical surface and altered cytoplasmic contents (Fig. 1a). Golgi apparati occupied large areas in the apical domain of the supporting cells, and a large number of microtubules were present. The heterologous junctional complexes with the neighboring hair cells were unaltered and included

Fig. 1. Micrographs of TEM sections of supporting cells, cut perpendicular to the reticular lamina two days after noise exposure. (a): The apical domain of a supporting cell is located between two surviving hair cells (curved arrow points to a stereocilium of one hair cell). Several Golgi complexes $(G)$ and microtubules (open arrow) are present in the supporting cell cytoplasm. The junctional complexes include adherens junctions (arrow) and, above them, tight junctions. Numerous microvilli extend from the apical surface of the supporting cell (arrow heads). (b): Supporting cell nuclei located near the basement membrane. The area of contact is flat and includes several indentations. (c): Expanded supporting cells occupy the lesion, where hair cells are missing. Two vertical (perpendicular to the reticular lamina) basal bodies (open arrow) are located near the luminal surface. Golgi complexes are abundant (arrow). Bars, (a) $250 \mathrm{~nm}$, (b) $500 \mathrm{~nm}$, (c) $2 \mu \mathrm{m}$. 


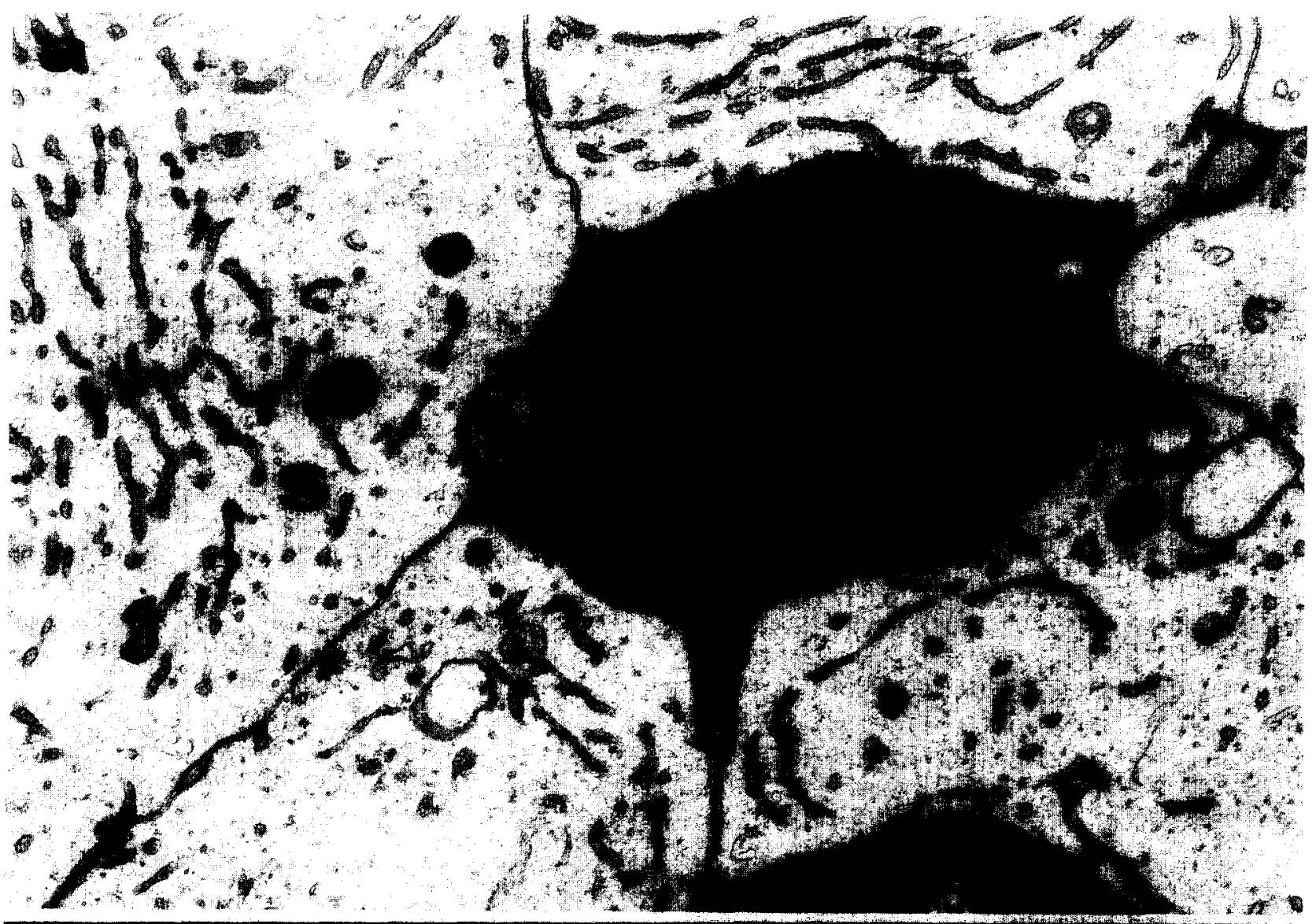


tight junctions and adherens junctions. Thus, loss of adjacent hair cells was not a prerequisite for a detectable response of supporting cells to trauma.

\section{Nuclear migration to the basement membrane}

The nuclei of many supporting cells were located in close proximity to the basement membrane. The part of the nuclei facing the basement membrane often appeared flattened and, at times, slightly indented (Fig. 1b). BrdU-specific immunoreactivity was detected in some of these nuclei, indicating that they replicated their DNA after the onset of the noise exposure (not shown). No cytoplasmic or cytoskeletal structures were associated with nuclei, regardless of nuclear position.

\section{Trauma-induced changes in cell shape and cytoplasmic organization}

In areas of hair cell loss, supporting cells were largely expanded. Basal bodies were commonly found in expanded supporting cells. Basal bodies were often oriented perpendicular to the reticular lamina (Fig. $1 \mathrm{c})$, but in some cases, they were oriented parallel to the reticular lamina (not shown). Golgi complexes were abundant in expanded supporting cells.

\section{Normal versus dividing supporting cells}

In control animals, sections cut parallel to the reticular lamina (about 3 microns beneath the luminal surface) revealed the body of a hair cell surrounded by 5 supporting cells (Fig. 2a). The membranes of the apposing cells were closely packed in homologous and heterologous junctions. Gap junctions (not shown) were restricted to homologous junctions in this tissue, as previously reported (Ginzberg and Gilula, 1979).

In noisc-cxposed chicks, dividing cells were found in the lesion. These cells were large and round and their DNA was condensed into mitotic chromosomes (Fig. 2b). Many of the dividing cells had membrane contact with a neighboring hair cell. These contacts were close, leaving no visible intercellular space between the two cells. These contact areas appeared smooth, with no protrusions or indentations on either side. In contrast, dividing cells had distinctive intercellular spaces between them and neighboring supporting cells in most areas, although areas of contact could be found. The intercellular spaces included protrusions that appeared like small filopodia, extending from both the dividing cells and their neighbors. Taken together, these data showed that the basolateral membrane of dividing cells was detached from surrounding supporting cells. Surviving hair cells were innervated by efferent nerve terminals (Fig. 2b), but no synapses were observed on dividing cells.

TEM analysis of sections at a plane perpendicular to the reticular lamina revealed that junctional complexes of dividing cells included tight junctions, adherens junctions, and, when the neighboring cell was a supporting cell, desmosomes (Fig. 3a). The apical membrane of dividing cells bordered the lumen (Fig. $3 a, b$ and d) and their basolateral domains were round (Fig. $3 b$ and d). The apical (luminal) membrane of dividing cells was very smooth, with very few microvilli. In contrast, the apical surface of expanded supporting cells that were not dividing exhibited a thick layer of microvilli (Fig. 3b and 1a).

The orientation of the mitotic division was determined in cross-sections and whole mount preparations. Surface analysis of whole mounts of the basilar papilla labeled for BrdU revealed several BrdU-labeled nuclei. The majority of these nuclei were paired, with the nuclei in a given pair located very close to one another, at a similar focal plane and parallel to the reticular lamina (Fig. 3c). Cross-section of plastic embedded material revealed that, in telophase, chromosomes moved parallel to the reticular lamina (Fig. 3d). This orientation of the chromosomes was also visible with fluorescence analysis of whole mounts of the basilar papilla during anaphase (Fig. $3 \mathrm{e}$ ).

The organization of the cytoplasm and the intercellular contacts of dividing cells was analyzed with TEM. Spindle microtubules and chromosomes were the only prominent organelles observed (Fig. $4 a$ and b). OIganelles such as Golgi complexes and endoplasmic reticulum were not conspicuous. The membranes of dividing cells included several cytoplasmic protrusions (Fig. 4a), but gap junctions were not observed. Basal bodies (centrioles) were present at the poles of the mitotic spindle (Fig. 4b).

\section{Conclusion and interpretation of the data}

The results reported here were collected from still micrographs, taken at one time-point after noise exposure. To elucidate the temporal sequence of cell cycle in the basilar papilla from the 'frozen-time' images, we

\footnotetext{
Fig. 2. TEM section cut parallel to the reticular lamina approximately $3 \mu \mathrm{m}$ beneath the luminal surface in a control animal (a) and noise-exposed animal (b). (a): A hair cell (H) is surrounded by 5 supporting cells ( $\mathrm{S}$ on one of them). No spaces are found between the membranes of apposing cells. Homologous (supporting cell-supporting cell) junctions appear similar to the heterologous (supporting cell-hair cell) junctions. (b): A large and round dividing cell in the lesion, with anaphase chromosomes (arrow). The dividing cell borders a hair cell (H) and several supporting cells $(S)$. The contact with the hair cell is close, with no intercellular space and no protrusions. Contacts with other supporting cells have conspicuous intercellular space and exhibit filopodia-like protrusions. An efferent nerve terminal (open arrow) synapses on the hair cell. Bar, $1 \mu \mathrm{m}$ for (a) and (b).
} 


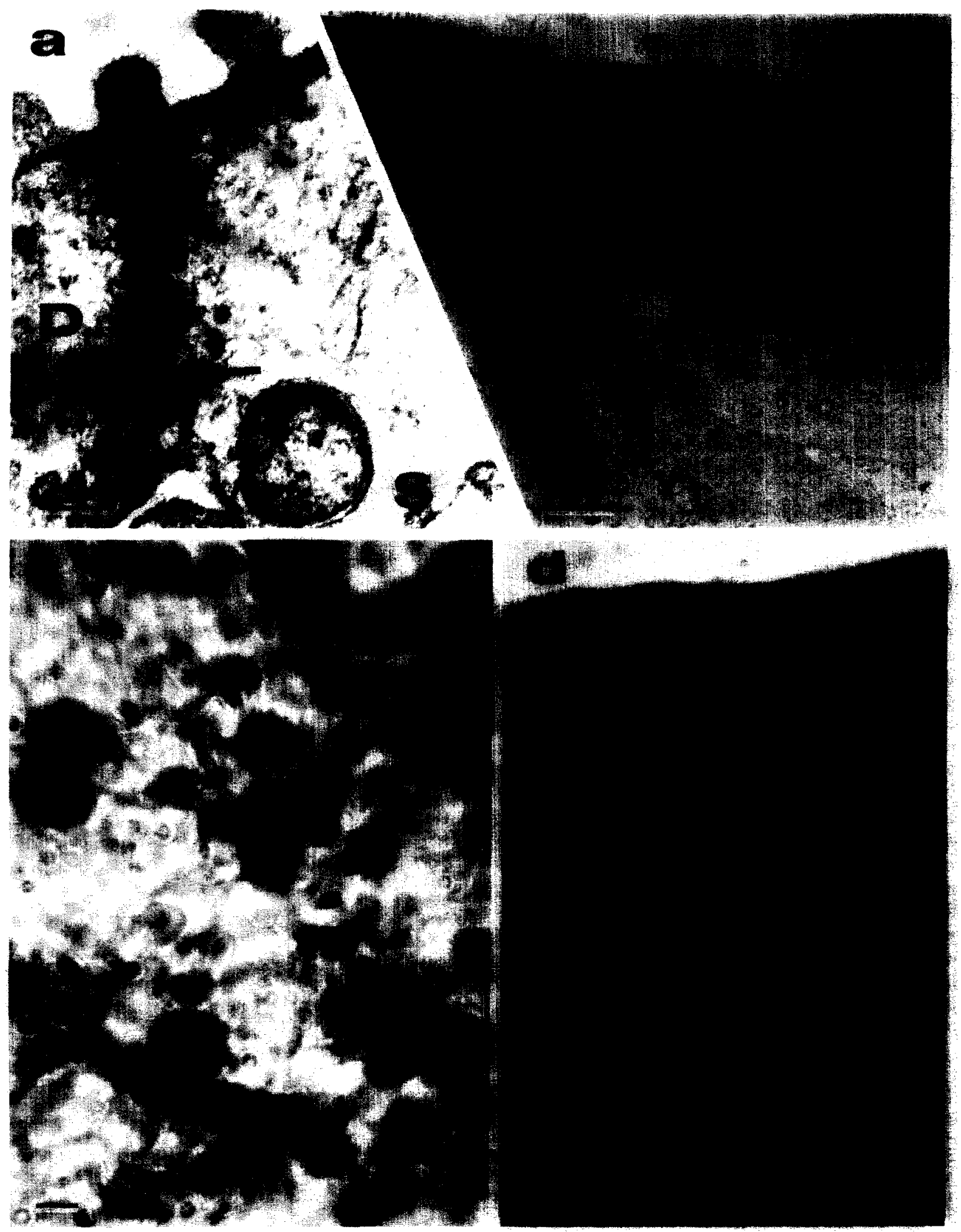


had to rely on comparisons to the structure of the normal basilar papilla and on data describing cell cycles in other systems. Fig. 5 schematically presents the major findings in this work, reconstructed into a temporal sequence based on the general understanding of proliferation in other epithelia. Our data describe morphological features of supporting cells during stages of transdifferentiation, including cellular shape, nuclear location, intercellular contacts, plane of mitosis and apical surface morphology. Our temporal sequence reconstruction suggests that the pattern of cellular proliferation in the basilar papilla after acoustic trauma is similar to interkinetic nuclear migration, previously described in other systems.

\section{Discussion}

Although all the data were collected on the second day after exposure, we report here data on different stages in the temporal sequence of cell cycle of supporting cells in the basilar papilla. The simultaneous presence of cells in S-phase, M-phase and post-mitotic cells indicated that supporting cells proliferation was asynchronous.

Nuclear migration, as described during early neurogenesis (Sauer, 1935) seems to occur during cell cycle in the avian auditory epithelium. In order for this migration to take place, the cell needs to know the location of the basement membrane and the nucleus must translocate. The reasons for this migration and the mechanisms that regulate and execute it need to be elucidated. Once near the basement membrane, the nucleus changes its shape and becomes flat against the basement membrane, possibly increasing the contact area with the membrane. In most epithelial cells, basal cell populations are located close to the basement membrane where they undergo S-phase (cornea, skin, respiratory, olfactory, glandular etc.). We now demonstrate that this location for S-phase is preferential also for the regenerating auditory epithelium, where basal cells have not been identified. It is likely that information is exchanged between the nucleus and the basement membrane and/or extracellular matrix. This may facilitate the influence of the extracellular matrix on DNA replication in supporting cells, although it is not clear why the nucleus needs 10 be so close to the basement membrane. Nuclei detach from the basement membrane and move toward the apical pole of the cell in order to divide near the lumen. The mechanisms that perform these highly coordinated spatial-temporal changes in nuclear position and shape are unknown, although the changes are common to cellular proliferation in most epithelial tissues.

Our observations that dividing cells in the traumatized basilar papilla are round and detached from the basement membrane are based on analysis of hundreds of sections taken from more than 20 dividing cells. In analyzing this material we have never observed protrusions towards the basement membrane. From this we conclude that before division, the nucleus migrates toward the apical pole and the basal pole detaches from the basement membrane in preparation for rounding up and dividing. Serial sectioning of dividing cells will be necessary to prove this point beyond doubt.

Findings that (a) dividing cells had luminal contact and (b) they were connected to neighboring supporting cells with desmosomes support the notion that dividing cells are most probably supporting cells, because in this tissue, only supporting cells have luminal surface and desmosomes. Dividing supporting cells often bordered surviving hair cells. It is possible that an exchange of information occurs between dividing cells and surviving hair cells. However, dividing cells were also found in areas without hair cells, suggesting that the presence of contact with a hair cell is not necessary for division to occur. This corroborates well with the finding that regeneration can occur after severe acoustic trauma involving total hair cell loss in the lesion (Cotanche, 1987a).

Once supporting cells were rounded up and dividing, the only conspicuous organelles that appeared to be present in their cytoplasm were chromosomes, spindle microtubules and centrioles. Although cell division can occur without centrioles (see Calarco-Gillam et al., 1983 for review), the presence of centrioles is strongly correlated with the establishment of polarity and the plane of division. Centrioles in non-dividing supporting cells are present in the apical pole as basal bodies. In order for centrioles to be present at the two poles of the spindle, they must either divide and migrate or be synthesized de novo. Future experiments will be neces-

\footnotetext{
Fig. 3. TEM sections cut perpendicular to the reticular lamina (a and b) and light microscope analysis of BrdU labeled whole mount (c), plastic section (d) and DNA-specific labeled whole mount (e), 2 days after the trauma. (a): The junctional complexes between a dividing cell (D) and a non-dividing supporting cell (S) include complexes of tight junctions, adherens junctions and desmosomes (arrow). (b): A round dividing cell with chromosomes (arrow). The apical surface contacts the lumen and basolateral borders do not reach the basement membrane. The luminal surface has very few microvilli. The luminal surface of an adjacent non-dividing supporting cell (S) has a dense microvillar cover. (c): Six pairs of nuclei with BrdU label located at a similar focal plane just beneath the reticular lamina. (d): A dividing cell in telophase. with the long axis of the spindle parallel to the reticular lamina. The furrow of the contractile ring is seen, in preparation for cytokinesis. (e): Two sets of chromosomes in an anaphase cell, with the long axis of the spindle parallel to the reticular lamina. Bars, (a) $100 \mathrm{~nm}$, (b) $2 \mu \mathrm{m}$, (c) $5 \mu \mathrm{m}$, (d) $10 \mu \mathrm{m}$, (e) $5 \mu \mathrm{m}$.
} 


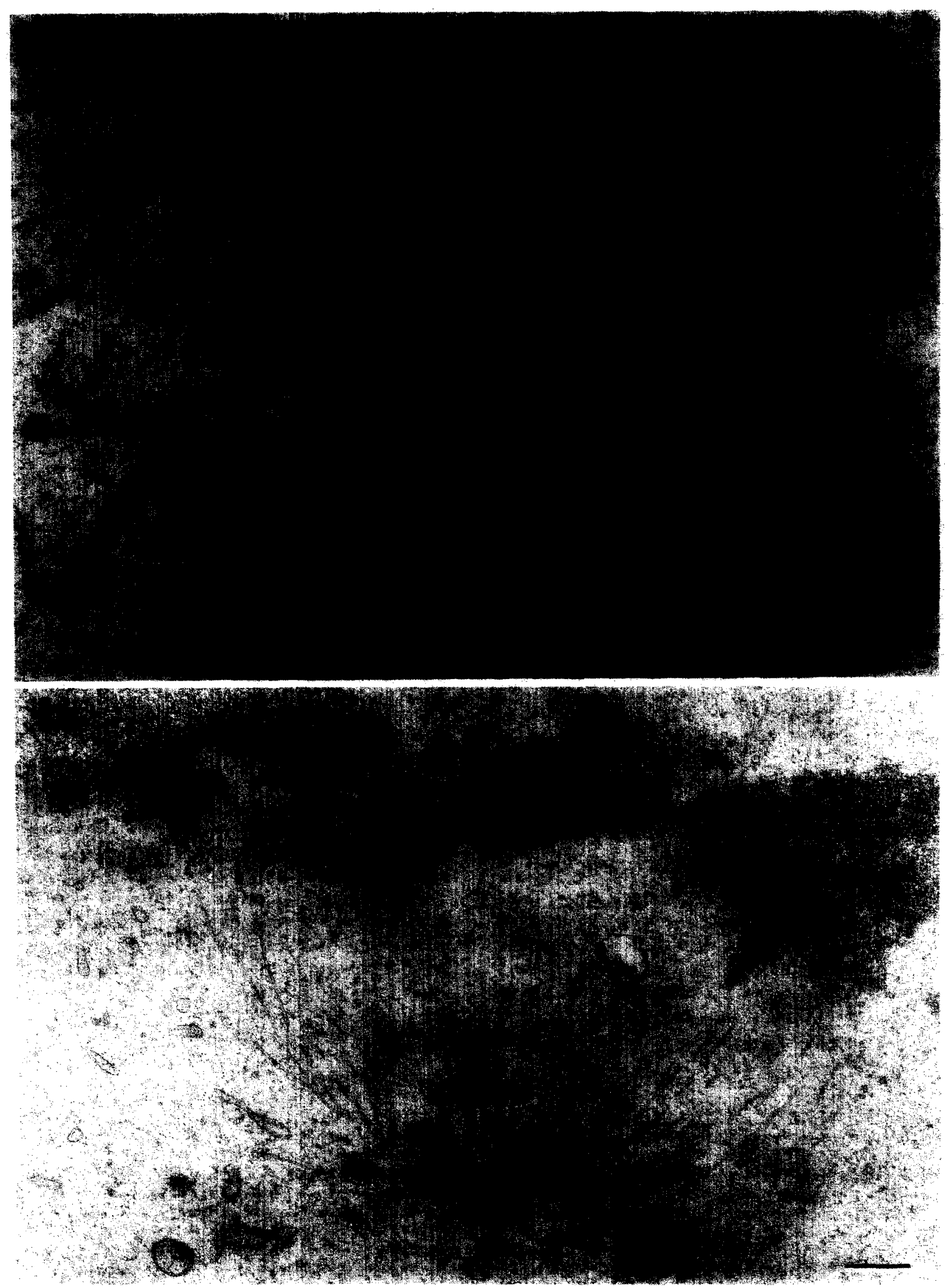


sary to determine how centrioles arrive at their sites in mitosis. Based on the frequent occurrence of basal bodies in expanded supporting cells, we speculate that the number of centrioles increases in supporting cells after trauma. Centriolar proliferation may temporally coincide with DNA replication, since no synthetic nuclear activity is necessary for centrioles to form or duplicate (Sluder et al., 1986). Moreover, centrosomal and nuclear events are regulated by separate pathways (Sluder et al., 1990). At present, it is not completely clear how basal bodies (or centrioles) receive information on the spatial orientation of the cell and its surroundings, but evidence that these organelles may be responsible for determining the orientation of cells in space has been provided (Albrecht-Buehler, 1992, 1994).

In these studies, we never saw a basal body or a centriole in a basal position (near the basement membrane) in supporting cells. This suggests that divisions with the long axis of the spindle perpendicular to the reticular lamina are uncommon or altogether absent during regeneration in this epithelium. In contrast, it is very likely that, during embryonic cytogenesis, divisions with the spindle perpendicular to the lumen (asymmetric divisions) do occur, creating new hair cells while replenishing the progenitors, as proposed in studies of cultured chick embryos (Katayama and Corwin, 1993).

It is not surprising that Golgi apparati, cytoskeletal networks and endoplasmic reticulum were absent (or nearly absent) in dividing cells. During division, the major concern of these cells is to divide the chromosomes safely and correctly, whereas transcriptional activity is unnecessary. In contrast to the actual time of mitosis, supporting cells in the lesion, when not in mitosis, are very active. Among the functions they perform are rapid change in volume (Cotanche, 1987a; Cotanche and Dopyera, 1990; Marsh et al., 1990; Raphael, 1992; Raphael and Altschuler, 1992), synthesis and secretion of new tectorial membrane to replace damaged tectorial membrane (Cotanche, 1987b; Adler et al., 1993), and reorganization of the apical contour, which probably involves synthesis of new junctional material (Raphael, 1993). Thus, a transition from the highly metabolic state to the dividing state must occur in these cells in preparation for mitosis. Golgi complexes disappear, as do the long microtubules from the cytoplasm and the endoplasmic reticulum. It appears from our data that, like other cell types (Franke et al., 1983), supporting cells rapidly reorganize their cytoplasm and cytoskeleton in preparation for division.

While in mitosis, supporting cells are attached to their neighbors with apical junctional complexes, but they appear to have neither gap junctions nor basement membrane contacts. This suggests to us that any information that may be necessary to initiate and regulate division, originating from the basement membrane, extracellular matrix or neighboring cells, must be conveyed to the dividing cells prior to the onset of division. The luminal surface of dividing cells had very few microvilli, indicating that interaction with the luminal fluids may also be limited during division. The only morphological signs of possible interaction with the environment during division are the small filopodia extended to the basolateral extracellular space.

The paucity of microvilli at the luminal surface of dividing cells, in addition to having a physiological implication, is a finding that could be utilized as a marker for identifying mitotic cells with the use of scanning electron microscopy (SEM). SEM is a method that is restricted to providing information on the external (luminal) surface of the basilar papilla, but one that is nevertheless very valuable for quantitative purposes. The analytical power of SEM increases if the surface morphology is better correlated with the physiological state of the cells. Equipped with this finding, we re-examined previous works that employed SEM analysis of the basilar papilla after insult and recovery time similar to the present work, and found that some supporting cells in the lesion had fewer microvilli than others. For example, we speculate that the supporting cell with a thin layer of microvilli, shown in a SEM micrograph in a previous report (the large cell just to the right of the center in Fig. 5A of Raphael, 1993), was in mitosis at the time of fixation. Further analysis will be necessary before this marker can be used safely for identification of dividing cells.

The division of supporting cells in the basilar papilla is parallel to the reticular lamina. This is consistent with the finding of pairs of BrdU-labeled nuclei immediately beneath the luminal surface. Together with the findings on pairs of new hair cells (Raphael, 1993), these data strongly support our speculation that a division of one supporting cell may often lead to formation of two new hair cells. Since both daughter cells

\footnotetext{
Fig. 4. TEM sections cut perpendicular to the reticular lamina, showing dividing cells in the basilar papilla. (a): Chromosomes (arrow) and spindle microtubules (open arrow) occupy most of the dividing cell cytoplasm, and no other cytoskeletal structures are detected. A distinctive intercellular space is present between a dividing cell and its non-dividing neighbor. Filopodia-like cytoplasmic protrusions are seen in the intercellular space, often appearing like extracellular vesicles (arrow heads). (b): The cytoplasm of a dividing cell contains few assembled organelles except the mitotic apparatus. The chromosomes (arrow) are pulled by microtubules in the direction of the pole, where a centriole is present (curved arrow). Bar, $500 \mathrm{~nm}$ for (a) and (b).
} 
may maintain luminal surface throughout division, the need for cellular migration is minimized. It remains to be determined whether, and under what circumstances, divisions lead to formation of two supporting cells or to asymmetric progeny (one hair cell and one supporting cell). Furthermore, Stone and Cotanche (1994) have shown that a second phase of divisions may follow the first cycle, with some cells dividing more than once. It would be important to elucidate the position and orientation of daughter cells that start replicating immediately after the first division.

For the new pair of hair cells to be incorporated into the normal mosaic of the basilar papilla, the two cells should be separated by apical processes of supporting cells. It is not clear how this separation is accomplished in the basilar papilla, but it is likely that the two daughter cells are separated without losing contact with neighboring cells, while maintaining the integrity of the epithelial sheet, as elegantly described in the proliferating retinal pigment epithelium (Sandig and Kalnins, 1990).

In conclusion, our data confirm that cell proliferation in the basilar papilla after acoustic trauma follows the pattern of interkinetic nuclear migration, as previously described for early neurogenesis (Sauer, 1935). Supporting cells that usually reside in the basilar papilla maintain their luminal surface throughout the cell cycle. Their nuclei undergo $S$ phase adjacent or close to the basement membrane and later migrate apically for division. We speculate that the extracellular matrix in the basilar membrane interacts with the epithelium and contributes to regulation of the regeneration.
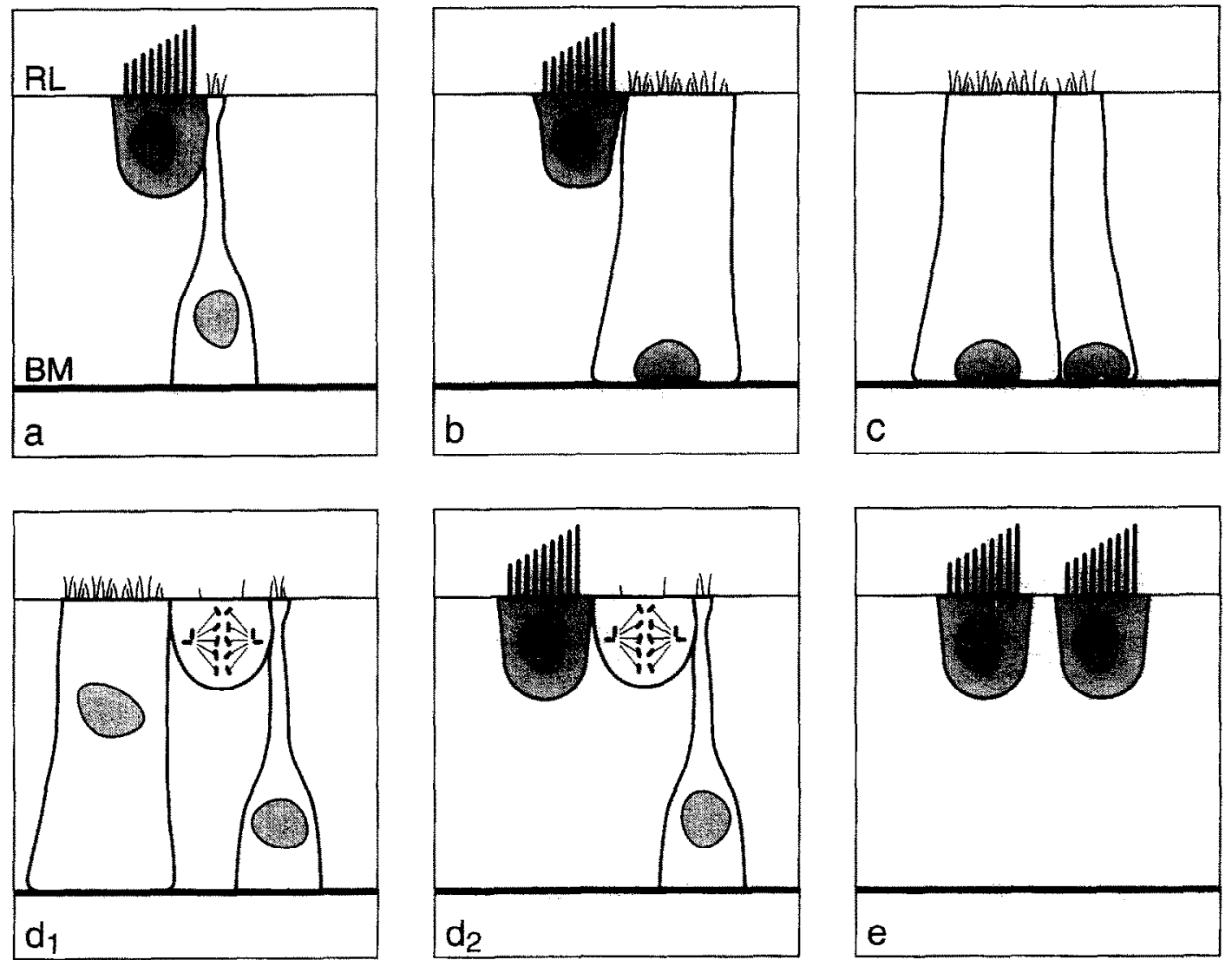

Fig. 5. Schematic of interkinetic nuclear migration in supporting cells of the basilar papilla, based on the data presented in this work and in Raphael (1992). (a): Normal appearance of neighboring hair cell and supporting cell. The hair cell is short and does not extend to the basement membrane. The supporting cell is long and slender, spanning the distance between the luminal surface (reticular lamina) and the basement membrane. Stereocilia extend from the luminal surface of hair cells and microvilli from that of supporting cells (RL-reticular lamina. BM-basement membrane). (b-c): After trauma, supporting cells expand, whereas hair cells either shrink (b) or degenerate (c). In either case, nuclei of supporting cells migrate to the region of the basement membrane for S-phase, while the apical surfaces of supporting cells maintain their dense microvillar cover. (d): The basal pole of supporting cells detaches from the basement membrane, and the cells round up near the luminal surface and divide. Mitotic cells may be surrounded by supporting cells (d1) or contact a hair cell (d2). The axis of the spindle is parallel to the reticular lamina. The luminal surface of dividing cells is smooth and nearly devoid of microvilli for the duration of the division. Dividing celis often, but not always, share apical junctions with surviving hair cells. (e): In some cases, one division can lead to generation of two new hair cells, separated by a supporting cell. 


\section{Acknowledgments}

We thank Dr. Donna M. Martin for valuable comments and Anat Sapan for excellent technical assistance. 'This work was supported by a NIDCD grant R01 DC01634.

\section{References}

Adler, H.J., Pole, C.P. and Saunders, J.C. (1993) Recovery of auditory function and structure in the chick after two intense pure tone exposures. Hear. Res. 71, 214-224.

Albrecht-Buehler, G. (1992) Rudimentary form of cellular "vision". Proc. Natl. Acad. Sci. USA. 89, 8288-8292.

Albrecht-Buehler, G. (1994) Cellular infrared detector appears to be contained in the centrosome. Cell Motil. 27, 262-271.

Calarco-Gillam, P.D., Siebert, M.C., Hubble, R., Mitchison, T. and Kirschner, M. (1983) Centrosome development in early mouse embryos as defined by an autoantibody against pericentriolar material. Cell 35, 621-629.

Cotanche. D.A. (1987a) Regeneration of hair cell stereociliary bundles in the chick cochlea following severe acoustic trauma. Hear. Res. 30, 181--196.

Cotanche, D.A. (1987b) Regeneration of the tectorial membrane in the chick cochlea following severe acoustic trauma. Hear. Res. 30, 197-206.

Cotanche. D.A. and Dopyera, C.E.J. (1990) Hair cell and supporting cell response to acoustic trauma in the chick cochlea. Hear. Res. $46,29-40$

Franke. W.W., Schmid, E., Wellsteed, J., Grund, C., Gigi, O. and Geiger, B. (1983) Change of cytokeratin filament organization during the cell cycle: selective masking of an immunologic determinant in interphase PtK2 cells. J. Cell Biol. 97, 1255-60.

Ginzberg. D.R. and Gilula, B.N. (1979) Modulation of cell junctions during differentiation of the chicken otocyst sensory epithelium. Dev. Biol. 68, 110-129.

Graziadei. P.P. and Monti Graziadei, G.A. (1985) Neurogenesis and plasticity of the olfactory sensory neurons. Ann. NY Acad. Sci. $457,127-142$.

Hirokawa. N. (1978) The ultrastructure of the basilar papilla of the chick. J. Comp. Neurol. 181, 361-374.

Jahnke, V., Lundquist, P.G. and Wersall, J. (1969) Some morphologi- cal aspects of sound perception in birds. Acta Otolaryngol (Stockh.) 67(6), 583-601.

Katayama, A. and Corwin, J.T. (1993) Cochlear cytogenesis visualized through pulse labeling of chick embryos in culture. J. Comp Neurol. 333, 28-40

Leblond, C.P. and Walker, B.E. (1956) Renewal of cell populations. Physiol. Rev. 36, 255-276.

Marsh, R.R.. Xu, L., Moy, J.P. and Saunders, J.C. (1990) Recovery of the basilar papilla following intense sound exposure in the chick. Hear. Res. 46, 229-238.

Raphael, Y. (1992) Evidence for supporting cell mitosis in response to acoustic trauma in the avian inner ear. J. Neurocytol. 21 $663-671$.

Raphael, Y. (1993) Reorganization of the chick basilar papilla following acoustic trauma. J. Comp. Neurol. 330, 521-532.

Raphael, Y. and Altschuler, R.A. (1992) Early microfilament reorganization in injured auditory epithelia. Exp. Neurol. 115, 32-36.

Sauer, F.C. (1935) Mitosis in the neural tube. I. Comp. Neurol. 6? $377-405$.

Sandig, M. and Kalnins, V.I. (1990) Reorganization of circumferential microfilament bundles in retinal pigment epithelial cells during mitosis. Cell Motil. 17, 133-141.

Sluder, G., Miller. F.J. and Rieder, C.L. (1986) The reproduction of centrosomes: nuclear versus cytoplasmic controls. J. Cell Biol. $103,1873-1881$.

Sluder, G., Miller, F.J., Cole, R. and Rieder, C.L. (1900) Protein synthesis and the cell cycle: centrosome reproduction in sea urchin eggs is not under translational control. J. Cell Biol. 110 , 2025-2032.

Stone, J.S. and Cotanche, D.A. (1992) Synchronization of hair cell regeneration in the chick cochlea following noise damage. I. Cell Sci. 102, 671-680.

Stone, J.S. and Cotanche, D.A. (1994) Identification of the timing of $S$ plase and the patterns of cell proliferation during hair cell regeneration in the chick cochlea. J. Comp. Neurol. 341, 50-57.

Stone, L.S. (1950) The role of retina pigment cells in regenerating neural retinae of adult salamander eyes. J. Exp. Zool. 113, 9-31.

Stroeva, O.G. and Mitashov, V.I. (1983) Retinal pigment epithelium: Proliferation and differentiation during development and regen eration. Int. Rev, Cytol. 83, 221-293.

Takasaka, T. and Smith, C.A. (1971) The structure and innervation of the pigeon's basilar papilla. Ultrastruct. Res. 35, 2()-65.

Tanaka, K. and Smith. C.A. (1978) Structure of the chicken's inner ear: SEM and TEM study. Am. J. Anat. 153, 251-271. 